\title{
Dialisi peritoneale 2013: si può fare
}

\section{Ugo Teatini}

Direttore Nefrologia e Dialisi, A.O. “G. Salvini”, Garbagnate Milanese (MI)

\begin{abstract}
Peritoneal dialysis 2013: We Can do it
Abstract. The use of peritoneal dialysis is showing a decrease in Italy. We here take into account the possible ways to reverse this trend, respecting the responsibilities of the different figures implicated in the management of the therapy of chronic renal failure (nephrologist, nurse, institution, patient).
\end{abstract}

Key words: Peritoneal dialysis, Nephrologist, Nurse, Patient

Conflict of interest: None.

Financial support: None.

Accettato: 23 Luglio 2013

Siamo nel 2013; la questione è: che spazio ha (o può avere) la dialisi peritoneale in Italia? Dati noti italiani, europei e mondiali ci fanno vedere un lento e costante decremento nel suo utilizzo. Si è provata la strada "istituzionale", con delibere o circolari regionali che "imponevano" ai centri dialisi di trattare con dialisi peritoneale circa il $20 \%$ dei pazienti che avevano bisogno di dialisi; ma, come in epoca manzoniana, sono state "grida" non ottemperate.

Più interessante si era dimostrata la via del contributo economico ai pazienti in trattamento peritoneale; le normative erano ben costruite; aveva iniziato la Sardegna, seguita poi da Piemonte, Sicilia e Puglia: ma anche questa esperienza non possiamo dire sia stata un successo, se si valutano i numeri di pazienti in queste regioni prima delle proposte di contributo ai pazienti domiciliari e dopo.

Io terrei conto di questi fattori:

- nefrologo

- infermiere

- paziente

- istituzione

Nefrologo: il nefrologo è importante per il consiglio terapeutico al paziente, ma lo è soprattutto tutto il team della Nefrologia e Dialisi; ma quello che è da ribadire è questo, a mio avviso: le Nefrologie che non offrono la possibilità terapeutica della dialisi peritoneale sono reparti a cui manca (dal punto di vista professionale) un pezzo di competenza; la dialisi peritoneale, invece, è da considerare come STRUMENTO nelle nostre mani per venire incontro alle esigenze che, in Italia, si esprimono mediamente nel $10-15 \%$ dei pazienti prevalenti.
Infermiere: anche qui l'esperienza ci dice che, laddove esiste un team infermieristico che svolge colloqui con i pazienti prima di iniziare la dialisi, illustrando le possibili opzioni terapeutiche di sostituzione della funzione renale, la dialisi peritoneale è ben sviluppata.

Paziente: teniamo conto che la scelta finale è del paziente, per cui corretta e tempestiva informazione e accoglienza sono fondamentali per una scelta consapevole; varie survey (non ultima quella fatta in Lombardia lo scorso anno) ci fanno vedere che pazienti informati scelgono la dialisi peritoneale in misura molto maggiore di quanto stia accadendo oggi.

Istituzione: esistono progetti di deospedalizzazione della dialisi, attraverso la possibilità della dialisi peritoneale assistita, attualmente al vaglio di due ASL lombarde; i concetti principali sono di poter utilizzare il capitolo di spesa dei trasporti dei pazienti in emodialisi per assicurare l'assistenza di un operatore sanitario oppure di far istituire dalla regione una tariffa a sé per la dialisi peritoneale assistita; il centro dialisi si accerta del corretto addestramento del personale secondo le specifiche necessità cliniche del paziente. Senza una collaborazione reale con le istituzioni è difficile poter affrontare i problemi dei nostri pazienti, in particolare, la rimozione delle barriere socio-familiari e il fatto di assecondare la preferenza espressa dal paziente per la terapia domiciliare. Sottolineo che lo scopo primario è quello di andare incontro a un trattamento adeguato dei pazienti; poi, possiamo prendere in considerazione il vincolo finanziario, importante ma secondario: ma, anche da questo punto di vista, l'iniziativa sarebbe vantaggiosa per l'amministrazione regionale rispetto al "classico" trattamento di emodialisi. 


\section{Riassunto}

La dialisi peritoneale manifesta un decremento nella sua diffusione; vengono prese in considerazione le possibili vie per invertire questo trend, nel rispetto delle competenze dei vari attori implicati nella terapia sostitutiva della funzione renale (nefrologo, infermiere, istituzione, paziente).

Parole chiave: Dialisi peritoneale, Nefrologo, Infermiere, Paziente

Dichiarazione di conflitto di interessi: L'Autore dichiara di non avere conflitto di interessi.
Contributi economici degli autori: L'Autore dichiara di non aver ricevuto sponsorizzazioni economiche per la preparazione dell'articolo.

Indirizzo degli Autori:

Dr. Ugo Teatini

Direttore Nefrologia e Dialisi

A.O. "G. Salvini"

Via Forlanini 121

20024 Garbagnate Milanese (MI)

UTeatini@aogarbagnate.lombardia.it 